WS15-D01

\title{
DAS VSP Acquisition - Perspectives and
} Challenges

\author{
V. Lesnikov* (Total) \& C. Allanic (Total)
}

\section{SUMMARY}

The interest to the Distributed Acoustic Sensing (DAS) technology for borehole seismic data acquisitions is rapidly growing during the last several years. It opens up a lot of new opportunities of performing VSP acquisitions for time-lapse monitoring utilizing producer or injector wells. It can also be used in the high temperature, highly deviated or horizontal wells where deployment of conventional geophone tool arrays is always complicated. All of this could be done in a safe and cost effective manner with hundreds of downhole measurements recorded from the single shot at the surface.

Meantime the current limitations of the DAS VSP are also well known. High level of the noise floor, directivity pattern, attenuation of the signal with the length of the fiber cable, uncertainty of the depth determination are among the observed problems.

We discuss our current experience with DAS VSP recorded with the fiber cable deployed behind the production tubing and challenges for its widespread application, in particular deployment of the fiber cable and possible deepwater and subsea applications for which reliable underwater-mateable fiber-optic connectors and marinisation technology for DAS interrogators has yet to be developed. 


\section{Amsterdam '14}

The interest to the Distributed Acoustic Sensing (DAS) technology for borehole seismic data acquisitions is rapidly growing during the last several years. It opens up a lot of new opportunities of performing VSP acquisitions for time-lapse monitoring utilizing producer or injector wells. It can also be used in the high temperature, highly deviated or horizontal wells where deployment of conventional geophone tool arrays is always complicated. All of this could be done in a safe and cost effective manner with hundreds of downhole measurements recorded from the single shot at the surface.

Meantime the current limitations of the DAS VSP are also well known. High level of the noise floor, directivity pattern, attenuation of the signal with the length of the fiber cable and uncertainty of the depth determination are among the observed problems.

We discuss our current experience with DAS VSP recorded with the fiber cable deployed behind the production tubing and challenges for its widespread application, in particular deployment of the fiber cable and possible deepwater and subsea applications for which reliable underwater-mateable fiberoptic connectors and marinisation technology for DAS interrogators has yet to be developed. 